\title{
Optics in Curved Space
}

\author{
Vincent H. Schultheiss, ${ }^{1,2}$ Sascha Batz, ${ }^{1,2}$ Alexander Szameit, ${ }^{3}$ Felix Dreisow, ${ }^{4}$ Stefan Nolte, ${ }^{4}$ Andreas Tünnermann ${ }^{4}$ \\ Stefano Longhi, ${ }^{5}$ and Ulf Peschel ${ }^{2}, *$ \\ ${ }^{1}$ Max Planck Institute for the Science of Light, 91058 Erlangen, Germany \\ ${ }^{2}$ Institute for Optics, Information and Photonics, University Erlangen-Nuremberg, 91058 Erlangen, Germany \\ ${ }^{3}$ Physics Department and Solid State Institute, Technion, 32000 Haifa, Israel \\ ${ }^{4}$ Institute of Applied Physics, Friedrich-Schiller-University Jena, 07743 Jena, Germany \\ ${ }^{5}$ Dipartimento di Fisica, Politecnico di Milano, 20133 Milano, Italy
}

(Received 13 April 2010; published 27 September 2010)

\begin{abstract}
We experimentally study the impact of intrinsic and extrinsic curvature of space on the evolution of light. We show that the topology of a surface matters for radii of curvature comparable with the wavelength, whereas for macroscopically curved surfaces only intrinsic curvature is relevant. On a surface with constant positive Gaussian curvature we observe periodic refocusing, self-imaging, and diffractionless propagation. In contrast, light spreads exponentially on surfaces with constant negative Gaussian curvature. For the first time we realized two beam interference in negatively curved space.
\end{abstract}

DOI: 10.1103/PhysRevLett.105.143901

Introduction.-Regions of considerably curved spacetime remain inaccessible for a direct experimental investigation, but have been subject to extensive theoretical research. The interplay between space-time curvature and electromagnetic wave dynamics is claimed to provoke intriguing effects like the generation of Hawking radiation [1] or the Unruh effect [2]. The extreme conditions necessary to witness these phenomena are not found in the vicinity of Earth. Consequently, since the work by Unruh [3] several approaches have promoted analog models of curved space-time, which share particular features and allow for an investigation of resulting phenomena in the laboratory. In this vein, systems like the Bose-Einstein condensate [4] and optical solitons in glass fibers [5] are interpreted as dynamical systems on a background representing an effective space-time metric.

On the other hand, the formalism of general relativity (GR) has become an appealing tool to cope with the manipulation of light. Transformation optics utilizes the covariant formalism to design novel devices by pointing out the equivalence in Maxwell's equations between spacetime curvature and a modulation of the refractive index of the transmitting medium [6-9]. This modulation is achieved experimentally by artificial metamaterials, which until now are very hard to assemble and are often connected to severe losses during the propagation. It was recently proposed to use metamaterials to mimic a nonflat space-time [10].

In our work we refrain from refractive index modulation. We choose a more direct, geometric approach to study the consequences of space curvature on the evolution of light. To this end we abandon one spatial dimension and investigate light propagation on specifically shaped twodimensional surfaces, as introduced in [11].

Geometry of surfaces.-Picture a surface $S$ embedded in three-dimensional Euclidean space. For every regular point
PACS numbers: 42.25.Fx, 04.40.-b, 42.25.Hz, 42.82.Et

$P$ on the surface two tangent circles with maximal and minimal radii $R_{1}$ and $R_{2}$ can be found. The Gaussian curvature $K$ at $P$ is then defined as $K=\kappa_{1} \kappa_{2}$, where $\kappa_{1,2}=1 / R_{1,2}$ are the principal curvatures. For tangent circles facing opposite sides of the surface, $K$ is negative. The extrinsic curvature $H$ is given by $H=\left(\kappa_{1}+\kappa_{2}\right) / 2$. Proper lengths on curved surfaces are determined by means of the two-dimensional metric tensor $\left(g_{i j}\right)$ according to $d s^{2}=g_{i j} d x^{i} d x^{j}$, where $i, j=x, z$ are coordinates parametrizing the surface of interest. The Gaussian curvature $K$ depends on neither the actual embedding nor on the topology of the surface, but on the metric $\left(g_{i j}\right)$ alone-and vice versa [12]. The extrinsic (or mean) curvature $H$, on the other hand, is not an invariant with respect to the topology.

When neglecting polarization effects, the evolution of monochromatic light bound to an arbitrarily shaped twodimensional layer can be described by the scalar Helmholtz equation $[11,13]$

$$
\left(\Delta_{g}+k^{2}\right) \Psi=-\left(H^{2}-K\right) \Psi .
$$

Here $k=2 \pi n_{0} / \lambda$ is the wave number of light with wavelength $\lambda$, propagating in a material with refractive index $n_{0}$. All the experiments in this work were performed at $\lambda=$ $633 \mathrm{~nm}$. The scalar formulation is justified by the experiments, which show no dependence on the polarization. The covariant Laplacian $\Delta_{g}=(1 / \sqrt{g}) \partial_{i} \sqrt{g} g^{i j} \partial_{j}$, with $i, j=$ $x, z$, is given as a function of the metric tensor elements $g_{i j}$ and $g=\operatorname{det}\left(g_{i j}\right)$, and thus depends on the Gaussian curvature only. [The explicit dependence for macroscopically curved surfaces can be derived from Eq. (2) or found in [11].]

Influence of the extrinsic curvature.-As an example for the influence of the topology of the surface, represented by the extrinsic curvature $H$, we studied light propagation in a microscopically undulated waveguide [see Fig. 1(a)]. It 
was realized via the femtosecond direct-writing method [14]. High power femtosecond pulses are focused into bulk fused silica yielding an increase of the material's refractive index in the focal region [15,16]. Because of the use of a special fused silica with a high content of hydrogen, the writing process causes a massive foundation of nonbridging oxygen-hole color centers in the waveguide region. When excited by the guided beam, their fluorescence is imaged by scanning a microscope objective along the sample. For a detailed description of excitation and detection procedures, see, e.g., [17].

The manufactured waveguide has a sinusoidal cross section and consequently a vanishing Gaussian, but noticeable extrinsic curvature varying periodically in the transverse direction. With an undulation amplitude of $4 \mu \mathrm{m}$, an undulation period of $30 \mu \mathrm{m}$, and a thickness of $10 \mu \mathrm{m}$, the extrinsic curvature $H$ is 2 orders of magnitude smaller than $k$. This is comparable with a refractive index change on the order of $10^{-5}$, which as known from nonlinear optics influences the diffraction behavior considerably [18]. Having no Gaussian curvature, the metric of flat space is reproduced and $\Delta_{g}$ becomes the common Laplacian. Only $H^{2}$ remains acting like an effective potential in the wave equation. The experimentally observed diffraction pattern [Fig. 1(c)] is in agreement with simulations based on Eq. (1) [Fig. 1(d)]. It reveals that light does not smoothly diffract as in flat space [Fig. 1(b)]. The diffraction pattern resembles that occurring in a periodic lattice [19], which now arises from the periodicity of $H^{2}$ only. The extrinsic curvature realizes thus a kind of topological photonic crystal [20].

Influence of the intrinsic curvature.-For macroscopic radii of curvature the influence of the right-hand side of
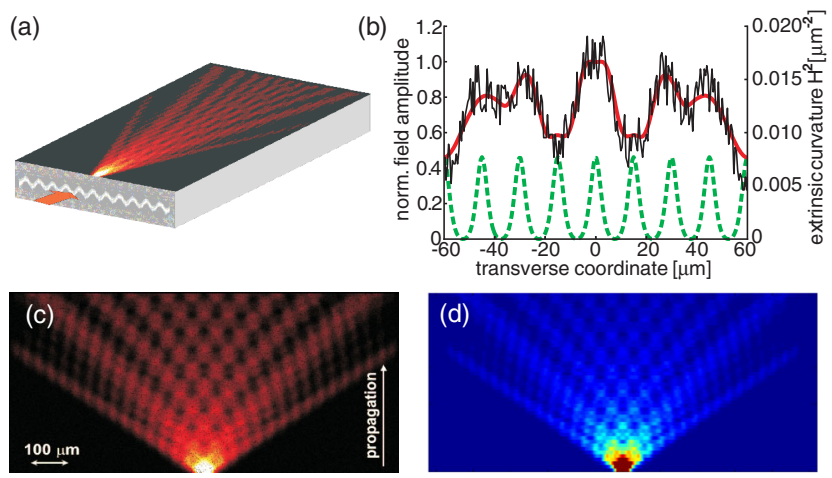

FIG. 1 (color online). The cross section in (a) is a microscope image of the undulated waveguide. (b) Comparison between experiment (solid black line) and simulation [solid gray (red) line]. Values are taken at half the total propagation distance displayed in (c) and (d). The dashed gray (green) line represents $H^{2}$, serving as an effective potential. (c) Directly monitored light propagating in the layer. The initial beam width $\sigma_{0}$ is about $10 \mu \mathrm{m}$ and the total propagation length is $25 \mathrm{~mm}$. Clearly the nonuniform diffraction is visible, resulting from the variation of the extrinsic curvature $H$. (d) Simulation of (c) based on Eq. (1).
Eq. (1) can be neglected, since $k^{2}$ dominates over $H^{2}$ and $K$. The Gaussian curvature $K$ still affects the beam evolution via the covariant Laplacian on the left-hand side. For convenience and as a natural generalization of flat Euclidean space, we restrict ourselves to rotationally symmetric surfaces of constant Gaussian curvature now. From the point of view of confined light beams, these surfaces are highly symmetric, namely, locally invariant under translation and rotation. Therefore, they are an optical realization of the cosmological principle, which states that space is homogenous and isotropic. This feature makes optics on curved surfaces a powerful tool for beam shaping, since it is in strong contrast to photonic crystals or structures with graded refractive index, where there is always a preferred direction involved in the design.

One has to be cautious when using the covariant formalism of GR, because any statement must be treated in the light of the chosen coordinates. Since we are dealing with rotationally symmetric surfaces only, we conveniently chose an orthogonal set of coordinates varying in proper lengths along the contour and the rotation angle of the solids of revolution (see [11]). However, while all equations must be understood for propagation along a certain direction, the physics is rotationally invariant and the stated evolution holds for other directions, too. Two solids with negatively curved surfaces were fabricated to allow for equal propagation lengths in different directions and to emphasize that equivalence [see insets of Figs. 4(a) and 4(c)].

Depending on the sign of Gaussian curvature, the metrics in the chosen set of coordinates read [11]

$$
d s^{2}=d x^{2}+\cos _{q}^{2}(x / R) d z^{2},
$$

where we introduced the notation $\cos _{1}=\cos , \cos _{-1}=$ cosh, with $q=\operatorname{sgn}(K)$ (analog for $\sin _{q}$ ). Here $R=$ $1 / \sqrt{|K|}$ is the geometric mean radius defined by the Gaussian curvature $K$. As long as a paraxial approximation can be applied, Gaussian beams maintain a Gaussian shape even in the presence of curvature. Analytical expressions describing the evolution of the beam width $\sigma$ with respect to the propagation length $z$ are [11]

$$
\sigma_{q}(z)=\sigma_{0} \sqrt{1-q\left(1-q \sigma_{S}^{4} / \sigma_{0}^{4}\right) \sin _{q}^{2}(z / R)},
$$

where $\sigma_{0}$ is the initial beam width and $\sigma_{S}=\sqrt{R / k}$ is a characteristic beam width defined by the surface itself. Following Eq. (2), which relates proper lengths to coordinates, the beam evolution can be explained by a compression or stretching of the wave vector components parallel to the propagation direction for $x \neq 0$. This corresponds to a redshift and blueshift in GR for negatively and positively curved surfaces, respectively.

Different solids with macroscopically curved surfaces were produced by means of high precision diamond turning (see Fig. 2). The simplest solids with positively curved 

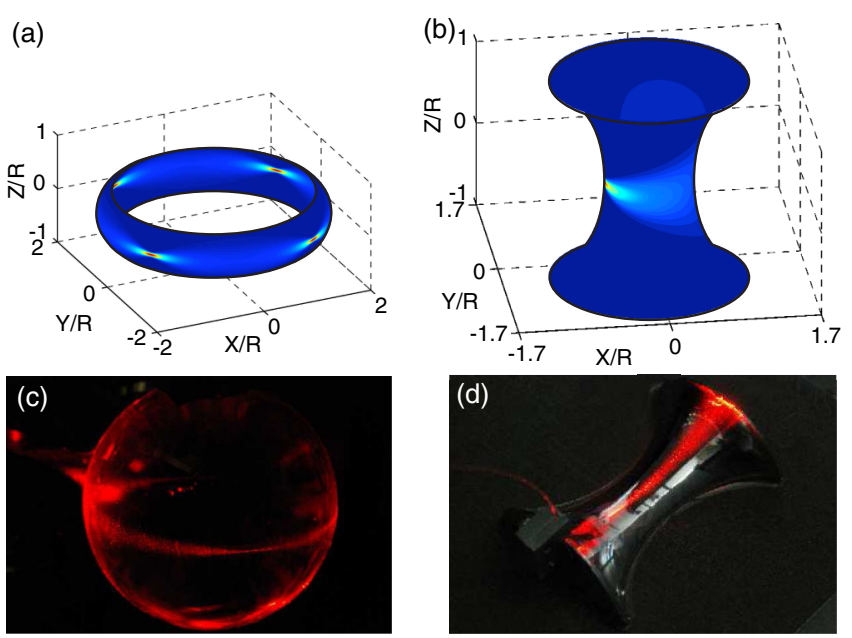

FIG. 2 (color online). (a),(b) Simulated propagation of an initial Gaussian beam on a positively and negatively curved surface. Refocusing on the bulge occurs after the same propagation length as on a sphere with equal $K$. The beam refocuses 4 times during propagation, for the arc length was chosen to be twice as long as that of the sphere. (c),(d) Experimental realizations of light propagation on a sphere and a hyperbolic surface. Since the beam evolution does not depend on the propagation direction, the same exponential spreading is observed in (b) and (d).

surfaces are spheres. For our experiments we used spheres and half-spheres which were made of BK7 glass. They had a radius of $R_{0}=R=1.25 \mathrm{~cm}$ representing a Gaussian curvature of $K=0.64 \mathrm{~cm}^{-2}$. In the case of the half-sphere a laser beam was impinging on the end face close to the edge. To couple light into the full sphere a coupling prism (SFL11 glass with $n_{0}=1.785$ ) was used, aided by a small drop of immersion liquid $\left(n_{0}=1.638\right)$ to increase coupling efficiency and allow for a wider range of initial beam widths at the surface. The beam is then guided inside the glass tangent to the surface by total internal reflection. A camera was rotated around the sample to detect the scattered light. After removing aberrations inevitably caused by imaging a curved surface, all camera images were composed to a single figure.

When launching a highly focused beam $\left(\sigma_{0} \approx 1 \mu \mathrm{m}\right)$ we observe a refocusing after a distance corresponding to half the circumference of a sphere [Figs. 3(a) and 3(b)]. This is in strong contrast to a flat surface. On a sphere the distance between two opposite points is always the same, no matter into which direction light travels. Hence, constructive interference must occur. As light cannot sense a macroscopic topology, the same phenomenon occurs on a bulge with equal $K$ after the same propagation length, which is then different from half a round-trip [see Fig. 2(a)]. When a broad beam is launched into the system, light already focuses after one-quarter of a round-trip, since now the evolution starts after $1 / 4$ of the oscillation period [Figs. 3(c) and 3(d)]. Still, the initial light distribu-
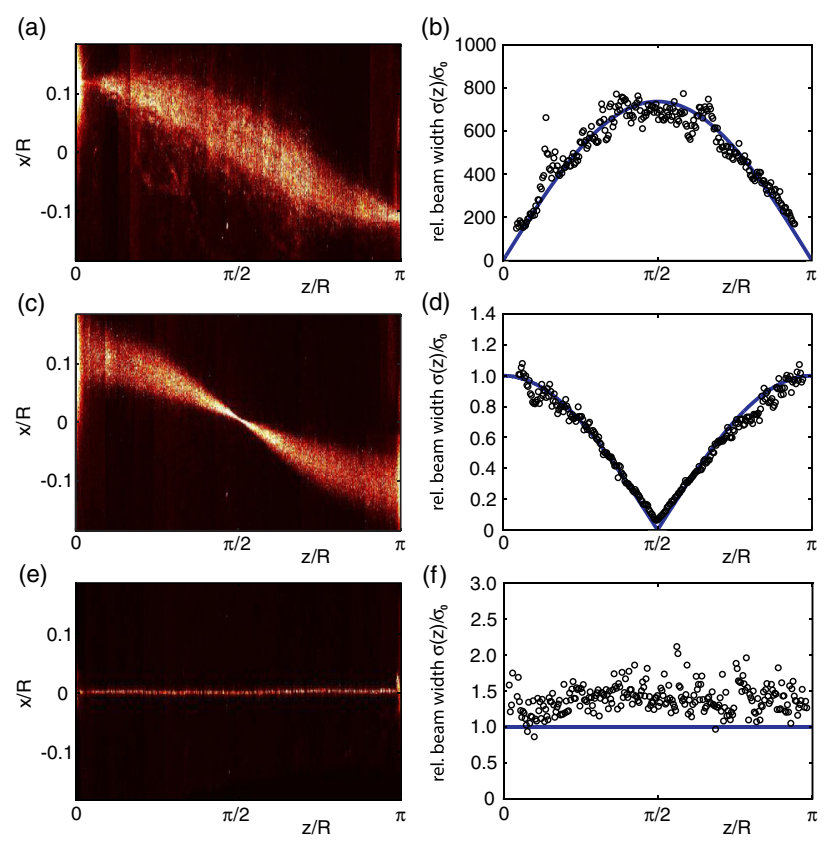

FIG. 3 (color online). Diffraction of a beam on a positively curved surface $(R=1.25 \mathrm{~cm})$. Propagation on the sphere for (a) a focused beam $\left(\sigma_{0}=1.1 \pm 0.1 \mu \mathrm{m}<\sigma_{S}\right)$, (c) a broad beam $\left(\sigma_{0}=561.3 \pm 69.2 \mu \mathrm{m}>\sigma_{S}\right)$, and (e) the stationary width $\left(\sigma_{0}=37.4 \pm 4.2 \mu \mathrm{m}\right.$, whispering gallery mode). The ecliptic in (a) and (c) is due to the camera being turned out of the equatorial plane to avoid disturbing stray light from the back side of the sphere. (b),(d),(f) Measured evolution of the beam width (circles) from a column-wise Gaussian fit of (a), (c), and (e), compared to the corresponding theoretical prediction [gray (blue) lines] based on Eq. (3). The values reveal a resolution limit of the measuring method.

tion is exactly imaged after half the circumference of a sphere. This imaging property of positively curved surfaces has in the past inspired some applications in radar techniques [21] and ray optics based geodesic lenses [22]. It is also reminiscent of that found in materials with special refractive index profiles (like Maxwell's fish-eye lens [23], which emulates the properties of a sphere in flat space). As the field evolution depends on the shape of the initial field, the natural question arises of whether or not an intermediate beam width exists for which light propagates without diffraction. Following Eq. (3) a whispering gallery mode is excited for a beam width $\sigma_{0}=\sigma_{S}$ [Figs. 3(e) and 3(f)]. Note that our system is highly symmetric. In contrast to, e.g., photonic crystals [24], diffraction free propagation can be realized for arbitrary frequencies and every propagation direction.

On negatively curved surfaces, light experiences an ever expanding space during propagation and every field distribution spreads exponentially. The bodies with negatively curved surfaces were fabricated from bulk aluminum and later covered with a high index immersion liquid $\left(n_{0}=\right.$ 1.518) of a few $100 \mu \mathrm{m}$ thickness acting as a waveguide. 

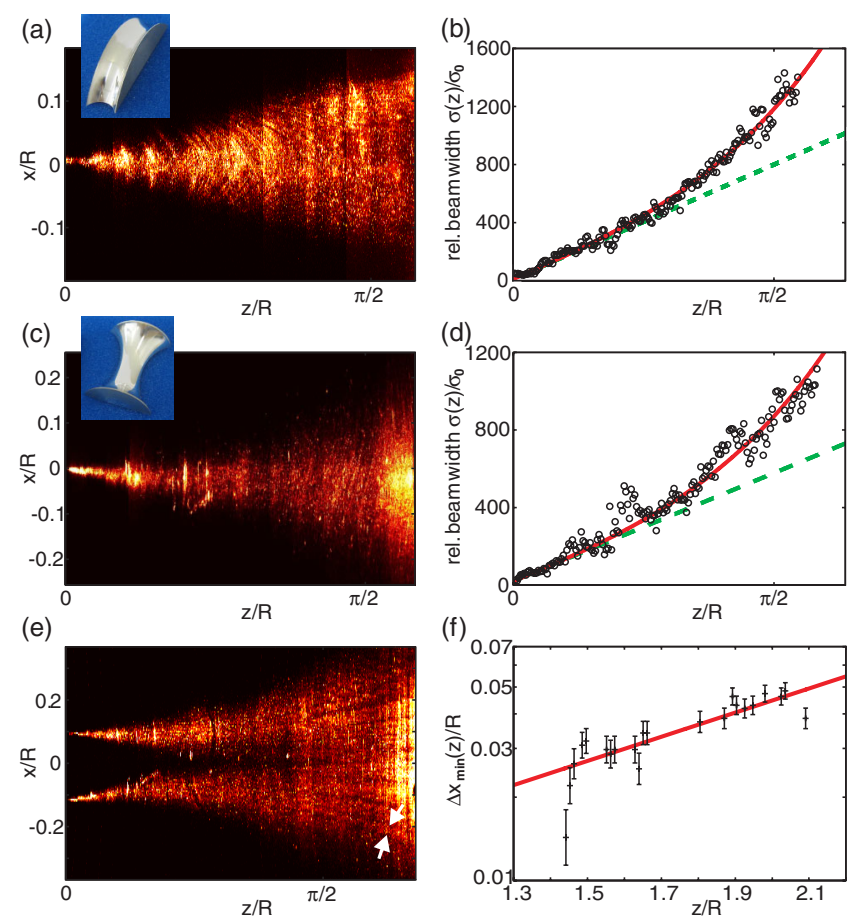

FIG. 4 (color online). Diffraction of beams on negatively curved surfaces $(R=1.25 \mathrm{~cm})$. Propagation along (a) rotation angle $\left(\sigma_{0}=1.3 \pm 0.1 \mu \mathrm{m}\right)$ and (c) contour $\left(\sigma_{0}=1.5 \pm\right.$ $0.2 \mu \mathrm{m})$. (b),(d) Measured evolution of the beam width (circles) of (a) and (c) compared to the theoretical prediction (solid red lines) based on Eq. (3), which is distinct from the evolution in flat space for the same initial excitations (dashed green lines). (e) Interference pattern of two beams launched in a distance $d=$ $2.2 \mathrm{~mm}$ on the same surface as in (c). (f) Tracking the distance between two adjacent intensity minima [white arrows in (e)] and fitting with an exponential function (solid red line) we obtain $R=1.24 \pm 0.16 \mathrm{~cm}$ in agreement with the nominal value.

The light was coupled into the film by using single mode glass fiber tips dipping into the film tangential to the surface. The scattered light was observed as in the case of the positively curved surface, skewing the images in such way that the final $x$ and $z$ direction represent our set of coordinates. To emphasize the equivalence of various embeddings in terms of beam evolution, we designed two different solids with negatively curved surfaces. Both surfaces had a constant negative Gaussian curvature of $K=$ $-0.64 \mathrm{~cm}^{-2}$ [see insets of Figs. 4(a) and 4(c)]. The experimentally observed exponential spreading is shown in Figs. 4(a)-4(d). Obviously, light experiences a repelling potential everywhere. Rays follow exponentially separating geodesics and no stationary state exists nor does any recovery occur. This phenomenon is even better visualized by launching two beams parallel in a distance $d$ and monitoring the resulting interference pattern. While in flat space the distance between interference fringes of two beams increases linearly for $z>d$ with the propagation length, on surfaces with negative Gaussian curvature interference fringes correspond to separating geodesics with the distance between minima $\Delta x_{\min }$ approximately scaling like $\exp (z / R)$ for $z / R>1$ [see Fig. 4(e)]. The experimentally observed fringe pattern is in good agreement with the theoretical prediction [Fig. 4(f)].

Conclusion.-We experimentally monitored the light evolution on curved surfaces and demonstrated how light is strongly influenced by the curvature, even if translational symmetry is still maintained. We showed that the extrinsic curvature matters only when light recognizes the surrounding space. Schemes and results presented in this work provide a first step towards a thorough experimental investigation. Also, nonlinear phenomena such as solitons can in principle be investigated in curved spaces [25]. Hence, the ideas proposed here open up many possibilities to improve the manipulation of light, construct analog models of general relativity, investigate critical orbits or even quantum billiards in curved spaces [26].

We thank the Cluster of Excellence Engineering of Advanced Materials of the University ErlangenNuremberg for supporting this work. We acknowledge further support by the German Academy of Science Leopoldina (Grant No. LPDS 2009-13).

*Corresponding author. ulf.peschel@mpl.mpg.de

[1] S. Hawking, Nature (London) 248, 30 (1974).

[2] W. G. Unruh, Phys. Rev. D 14, 870 (1976).

[3] W. G. Unruh, Phys. Rev. Lett. 46, 1351 (1981).

[4] C. Barcelo, S. Liberati, and M. Visser, Phys. Rev. A 68, 053613 (2003).

[5] T. G. Philbin et al., Science 319, 1367 (2008).

[6] L. D. Landau and E. M. Lifshitz, The Classical Theory of Fields (Butterworth-Heinemann, Oxford, 1980).

[7] U. Leonhardt and T. G. Philbin, Prog. Opt. 53, 69 (2009).

[8] J. Pendry, Nature (London) 423, 22 (2003).

[9] D. Schurig et al., Science 314, 977 (2006).

[10] D. A. Genov, S. Zhang, and X. Zhang, Nature Phys. 5, 687 (2009).

[11] S. Batz and U. Peschel, Phys. Rev. A 78, 043821 (2008).

[12] W. Kühnel and B. Hunt, Differential Geometry: CurvesSurfaces-Manifolds (American Mathematical Society, Providence, RI, 2005), 2nd ed.

[13] S. Longhi, Opt. Lett. 32, 2647 (2007).

[14] K. Itoh et al., MRS Bull. 31, 620 (2006).

[15] A. Szameit et al., Appl. Phys. B 82, 507 (2006).

[16] A. Szameit et al., Appl. Phys. Lett. 90, 241113 (2007).

[17] A. Szameit et al., Nature Phys. 5, 271 (2009).

[18] G. I. Stegeman and M. Segev, Science 286, 1518 (1999).

[19] H. S. Eisenberg et al., Phys. Rev. Lett. 81, 3383 (1998).

[20] A. Szameit et al., Phys. Rev. Lett. 104, 150403 (2010).

[21] S. B. Myers, J. Appl. Phys. 18, 221 (1947).

[22] G. C. Righini et al., Appl. Opt. 12, 1477 (1973).

[23] U. Leonhardt, New J. Phys. 11, 093040 (2009).

[24] R. Iliew et al., Appl. Phys. Lett. 85, 5854 (2004).

[25] S. Batz and U. Peschel, Phys. Rev. A 81, 053806 (2010).

[26] R. V. Jensen, Nature (London) 355, 311 (1992). 